\title{
sEMG Bias-driven Functional Electrical Stimulation System for Upper-Limb Stroke Rehabilitation
}

\author{
Yu Zhou, Student Member, IEEE, Yinfeng Fang, Member, IEEE, Kai Gui, Student Member, IEEE, Kairu \\ Li, Student Member, IEEE, Dingguo Zhang, Senior Member, IEEE, and Honghai Liu*, Senior Member, IEEE
}

\begin{abstract}
It is evident that the dominant therapy of functional electrical stimulation (FES) for stroke rehabilitation suffers from heavy dependency on therapists experience and lack of feedback from patients status, which decrease the patients' voluntary participation, reducing the rehabilitation efficacy. This paper proposes a closed loop FES system using surface electromyography (sEMG) bias feedback from bilateral arms for enhancing upper-limb stroke rehabilitation. This wireless portable system consists of SEMG data acquisition and FES modules, the former is used to measure and analyze the subject's bilateral arm motion intention and neuromuscular states in terms of their sEMG, the latter of multi-channel FES output is controlled via the sEMG bias of the bilateral arms. The system has been evaluated with experiments proving that the system can achieve $39.9 \mathrm{~dB}$ signal-to-noise ratio (SNR) in the lab environment, outperforming existing similar systems. The results also show that voluntary and active participation can be effectively employed to achieve different FES intensity for FES-assisted hand motions, demonstrating the potential for active stroke rehabilitation.
\end{abstract}

Index Terms-Functional electrical stimulation (FES), surface electromyography (sEMG), closed loop, bilateral arm bias, stroke, active rehabilitation.

\section{INTRODUCTION}

$\mathbf{S}$ TROKE is a common global health-care problem that has had serious negative impact on the life quality of the patients [1], [2]. Motor impairment after stroke typically affects one side of the body [2]. The main focus of stroke rehabilitation is the recovery of the affected neuromuscular functions and the achievement of independent body control [3]. Currently, there are many therapies applied to stroke rehabilitation, such as mirror therapy [4], repetitive task training [5], mental practice [6], robotics [7]. However, after completing standard rehabilitation, approximately $50 \sim 60 \%$ of stroke patients still suffer from some degree of motor impairment [8] and for the acute stroke patients with a marked impairment of function in one arm, even only about $14 \%$ of them will regain useful sensory-motor function [9]. One of the reasons is that most of therapies are open-loop and

Zhou, Gui, Zhang, and Liu are with the State Key Laboratory of Mechanical System and Vibration, School of Mechanical Engineering, Shanghai Jiao Tong University, Shanghai, 200240, China. (e-mail: hnllyu@sjtu.edu.cn; gkfxlt@foxmail.com; dgzhang@sjtu.edu.cn; honghai.liu@icloud.com).

Fang, Li and Liu are with the School of Computing, the University of Portsmouth, PO1 3HE, Portsmouth, UK. (e-mail: yinfengfang@gmail.com; kairu.li@port.ac.uk).

This work is supported by the National Natural Science Foundation of China (No. 51575338, 51575407, 51475427, 61733011) and the Fundamental Research Funds for the Central Universities (17JCYB03). passive. It excludes the active participation of subjects and decreases the neuromuscular activity and energy consumption, which leads to a reduction of rehabilitation efficacy [10], [11]. Thus, a closed loop active rehabilitation system is desired to improve the rehabilitation efficacy, which can not only detect patients' voluntary movement intention and muscle status, but also adjust the rehabilitation process in real time according to the biofeedback [12], [13].

The sEMG signals, containing a wealth of physiological information, have been widely applied in human-machine interfaces [14]. Many research efforts have been focused on applying EMG detected from the patients with stroke rehabilitation. For example, Edwards et al. [15] demonstrated the utility of EMG biofeedback applied to clinical treatment of stroke. Dipietro et al. [16] proposed detecting stroke patients' movement intention by monitoring EMG in selected muscles to trigger the assistance provided by the robot. $\mathrm{Hu}$ et al. [17] made use of EMG representing the affected hand muscles activation levels and their co-contraction indexes for a quantitative evaluation of motor functional recovery process in chronic stroke patients during wrist training. Cesqui et al. [18] proposed that EMG can be used to detect and interpret the normal and abnormal muscle patterns and provide feedback on their correct recruitment in stroke rehabilitation. These studies indicate that EMG signals can be used to represent neuromuscular pathological state and motion intention in stroke rehabilitation.

FES applies electrical pulse of low level in motor neurons to activate the skeletal muscle in attempt to restore the motor function and generate the desired motions for the paralyzed patients [19]. Since first used for foot drop rehabilitation by Liberson in 1960 [20], FES has become one of the important and effective treatments for stroke rehabilitation [21]. For example, Robbins et al. [22] reported the positive effect of FES to increase strength and increase gait speed for stroke patients. Sabut et al. [23] proposed that FES is a useful therapeutic tool combined with conventional rehabilitation program to improve the muscle strength of stroke patients. Alon et al. [24] concluded that upper extremity taskoriented training incorporating FES may improve the upper limb function rehabilitation efficacy during early stroke than it without FES, based on the experiment on fifteen patients. These studies verified the effectiveness of FES applied to stroke rehabilitation. However, the passive open-loop cycling FES therapies in those studies and clinic output the stimulation current at a predefined fixed model and are easy to induce muscle fatigue. The rehabilitation efficiency can be further 
improved when FES is applied with the EMG feedback, in which stimulation process is adjusted by the neuromuscular states in real time and muscle fatigue is alleviated because of the lower average stimulation intensity compared to the cycling constant FES. The EMG-driven closed-loop FES has been studied and shows an advantage over the cycling FES [25]. For example, Hong et al. [26] combined EMG-triggered FES with mental imagery training and observed an advantage over FES alone. Fujiwara et al. [27] proposed an EMG-based FES system for chronic stroke motor improvement, which can induce corticospinal plasticity. Zhang et al. [28] proposed an EMG-based closed-loop torque control strategy of FES, which provided satisfactory control accuracy and remarkable torque control performance.

However, problems still exist: 1) There are no objective and universal standards to evaluate the weakness degree of the affected muscle based on the EMG signals in those studies, because the EMG signals vary among people and also because of different processing methods; 2) Most existing commercial FES systems, such as the ParaStep system (Sigmedics. USA), the RehaStim2 (HASOMED GmbH, Germany), the NESS H200 (Bioness, USA), are open loop. Some other devices, such as the Compex Motion stimulator (Compex SA, Swiss), the WalkAide foot drop stimulator (NeuroMotion, USA), the NESS L300 (BIONESS, USA), are equipped with sensor interfaces for force-sensitive-resistors (FSR), accelerometers, push buttons. Although those systems are technically closedloop systems, the sensor input of them performs more like an on/off trigger which does not represent or make full use of users' movement intention or muscle status; 3) The use of separate FES and EMG devices to form the closed loop not only faces the communication and real-time problems between the subsystems but also makes the whole system cumbersome and time-consuming to attach and remove, which becomes one of the main reasons why EMG/FES closed-loop control strategies have not been widely used in clinical applications [19]. Thus, a state-of-the-art EMG/FES closed-loop active rehabilitation system is desired to solve the problems above.

The state-of-the-art rehabilitation system facilitating stroke recovery, especially for upper-limb stroke rehabilitation, drive the rehabilitation research frontier boundary forward and enhance life quality of stroke patients. This study proposes an attempt of a wireless multichannel EMG-FES system feedback by sEMG bias of bilateral arms for upper-limb stroke rehabilitation. The sEMG bias is introduced as a metric to trigger the FES module, and can be an index measuring to which degree a stroke patient recovered. The rest of this paper is organized as follows. The sEMG bias based system design is presented in Section II. The system evaluation is presented in Section III. Experiment is provided in Section IV. Finally, Section V concludes this study with discussions and future work.

\section{Multichannel FES TRigged by sEMG Bias}

This section presents the design of the sEMG bias based multichannel FES system. It should be noted that all the experimental procedures used in this study were approved by the SJTU School Ethics Committee and all subjects gave written informed consent and provided permission for publication of photographs for scientific and educational purposes.

Because the rehabilitation effect is enhanced when stimulation is associated with a voluntary attempt to move the limb [12], the stimulation intensity must be configured automatically in such a way that not only the desired movement task can be achieved but also the maximal possible volitional contribution is demanded to the patient. In general, the volitional EMG is obtained from non-paralysed muscles or stimulated muscles [29] and the threshold value or the envelope of the EMG signals are calculated to map the stimulation intensity statically and linearly [30]. However, the mapping relation has not been standardized and it is hard to quantify the muscles status, because of the EMG signals' high variability. Considering that EMG signals recorded from stroke subjects display high intersubject variability, we proposed that sEMG signals from the unaffected side of the stroke patients could be a reference standard to quantify the neuromuscular states of the affected side. The root mean square (RMS) value is selected as the sEMG feature in this study because it is a measure of the power of sEMG signal that is related to constant force and non-fatiguing contraction [31], [32]. As shown in Fig. 1, the volitional sEMG signals corresponding to the targeted mirror symmetry muscles of bilateral arms when doing the same action with the same effort are compared, and the root mean square (RMS) biases are the input of the FES controller. The FES controller is defined by the equation (1) and (2). The $Q$ in equation (2) is used to represent the weakness degree of the affected limb muscles and to map the stimulation intensity (amplitude or pulse width).

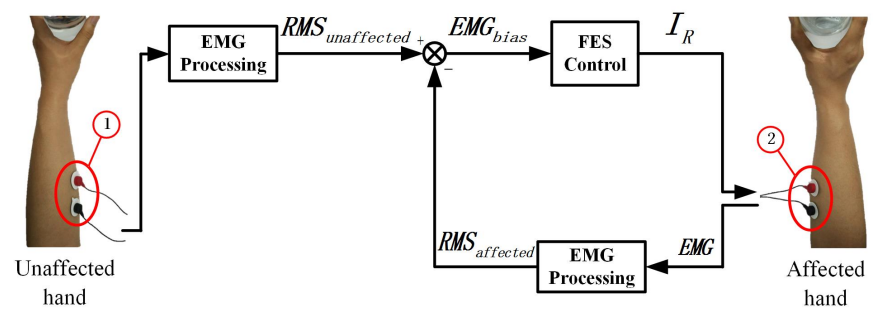

Fig. 1. Diagram for the sEMG bias-driven FES control strategy. 1 and 2 demonstrate the electrodes positions corresponding to the mirror symmetry muscles of the bilateral arms.

$$
\left\{\begin{array}{l}
E M G_{\text {bias }}=R M S_{\text {unaffected }}-R M S_{\text {affected }} \\
Q=\frac{E M G_{\text {bias }}}{R M S_{\text {unaffected }}}
\end{array}\right.
$$

where $R M S_{\text {affected }}$ and $R M S_{\text {unaffected }}$ are the RMS value of sEMG from the affected limb and the unaffected limb corresponding to the same movement.

$$
I_{R}=\frac{I_{\max }}{Q_{\max }-Q_{\min }} Q-\frac{I_{\max } Q_{\min }}{Q_{\max }-Q_{\min }}
$$

where $I_{R}$ is the realtime stimulation intensity. $I_{\max }$ is the maximum stimulation intensity. $Q_{\min }$ represents the threshold that the affected hand needs no stimulation to assist in performing the motion. While, $Q_{\max }$ represents the threshold for detecting the movement intention and triggering the FES. 


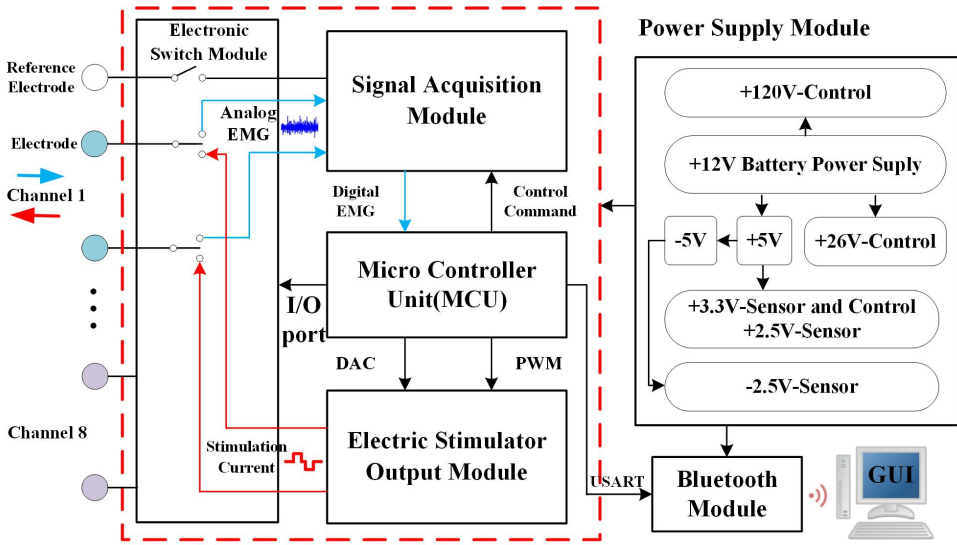

(a)

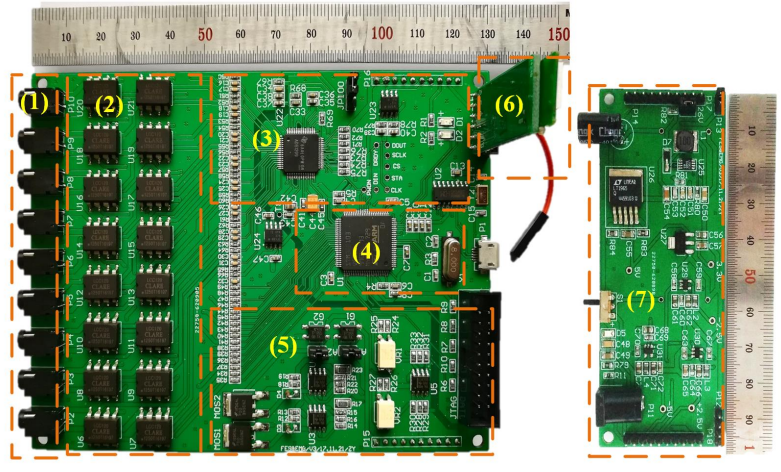

(b)

Fig. 2. The system design: (a) The architecture of the sEMG bias-driven FES system. (b) The prototype of the system: (1) the input and output interface, (2) Electronic switch module, (3) Signal acquisition module, (4) MCU, (5) Electric stimulator output module, (6) Bluetooth module, (7) Power supply module.

As shown in Fig. 2, the overall architecture is composed of seven parts: electronic switch module (ESM), signal acquisition module (SAM), microcontroller unit (MCU), electric stimulator output module (ESOM), power supply module (PSM), bluetooth module (BM) and graphical user interface (GUI). The channels of the system can not only transmit sEMG signals but also output stimulation currents. The ESM, controlled by MCU I/O port, is used to switch the connection between electrodes-to-ESOM and electrodes-to-SAM, and so that to protect SAM from the heavy current damage from ESOM.

\section{A. sEMG Bias Acquisition Module}

There are two steps for the sEMG bias acquisition, as shown in Fig. 3. First, the users are asked to perform the standard rehabilitation gestures, which will be used to train the affected hand later, with their unaffected hand in daily efforts. The sEMG RMS values of each channel, corresponding to the period when a certain movement is being hold on stably, are calculated and stored. Second, the users try to perform the same gesture with their affected hand, the sEMG RMS values corresponding to the same muscles as step 1 are calculated and compared with the relevant RMS values stored before. Thus, the sEMG biases for each channel of the system are generated in real time for FES control during corresponding gesture rehabilitation training.

There are various kinds of noises, such as baseline noise (electro-chemical noise from the skin-electrode interface and thermal noise due to the property of semiconductors), ambient noise (50 or $60 \mathrm{~Hz}$, power line noise) and movement artifact noise, can contaminate EMG signals. In order to extract clean sEMG from the noise background, the sEMG acquisition system should be with high common-mode rejection (CMRR) and high signal-to-noise ratio (SNR). In this study, we chose the ADC integrated chip ADS1299 (Texas Instruments) as the main component of SAM. The ADS1299, featured with CMRR $-110 \mathrm{~dB}$ and integrated with eight high-resolution simultaneous-sampling delta-sigma $(\triangle \Sigma)$ ADCs and eight

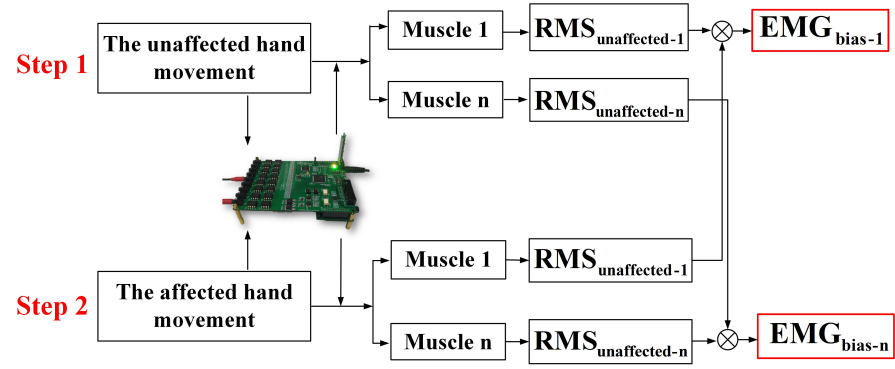

Fig. 3. Diagram for the sEMG bias acquisition. The step 1 is reference calibration, the step 2 is rehabilitation training. It should be noted that the gestures and the muscles are the same in step 1 and step 2.

low-noise programmable gain amplifiers (PGA), is designed for electroencephalogram (EEG) and biopotential measurements. That is to say, ADS1299 can not only restrain common mode noise but also amplify and digitize the raw sEMG signals.

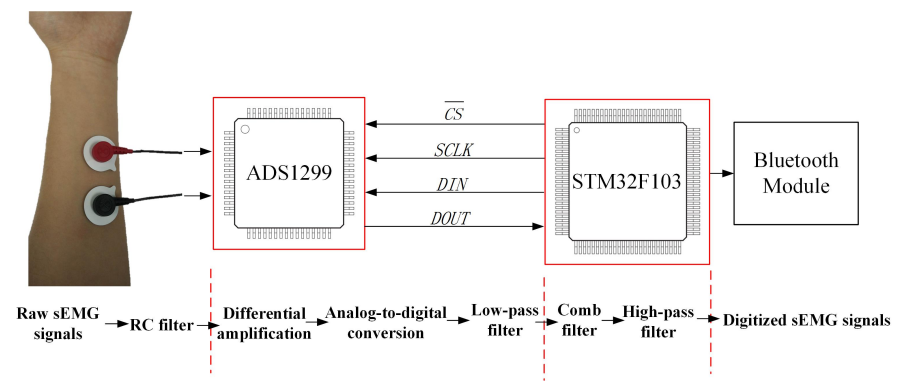

Fig. 4. The signal processing flow of SAM and MCU

As shown in Fig. 4, unlike the hardware filtering in [33], [34], the raw EMG signals are amplified, sampled and lowpass filtered in ADS1299 (the sampling rate is $1 \mathrm{kHz}$ ). The low-pass filer is a on-chip digital third-order sinc filter whose $\mathrm{Z}$-domain transfer function is (3). Then the high resolution digitized sEMG signals (frequency range: $0-500 \mathrm{~Hz}$ ) are transmitted into MCU (STM32F103VCT6, STMicroelectronics) 
through the serial peripheral interface (SPI). The comb filter (center frequency, $50 \mathrm{~Hz}$ and multiples thereof) and high-pass filter (cut-off frequency, $20 \mathrm{~Hz}$ ) towards the signals are realized in MCU to remove the baseline noise and movement artifact. The Z-domain transfer function of the comb filter is (4). The high-pass filter is a six-order Butterworth filter defined by (5) in Z-domain. Compared with the design in [33], the design in this study can not only extract clean sEMG but also save much space in PCB design.

$$
H(Z)_{\sin c}=\left|\frac{1-Z^{-N}}{1-Z^{-1}}\right|^{3}
$$

where $N$ is the decimation ratio of the filter.

$$
H(Z)_{c o m b}=\frac{i-1}{i}-\frac{1}{i} Z^{-T}-\frac{1}{i} Z^{-2 T}-\cdots-\frac{1}{i} Z^{-i T}
$$

where $T=f_{s} / f_{n}, f_{s}$ is the sampling frequency $(1 \mathrm{kHz})$ and $f_{n}$ is the power line noise $(50 \mathrm{~Hz}) ; i$ is the filter order, which determines the length of the previous signals being used to estimate noise.

$$
H(Z)_{\text {butterworth }}=\frac{\sum_{k=0}^{M} b_{k} Z^{-k}}{1-\sum_{l=1}^{N} a_{l} Z^{-l}}
$$

where $a$ and $b$ are coefficients; $N=M=7$.

\section{B. Functional Electric Stimulation Module}

The design for the output of the FES system is either constant-voltage, constant-current or a hybrid form [35]. Compared with constant-voltage, constant-current has the ad-

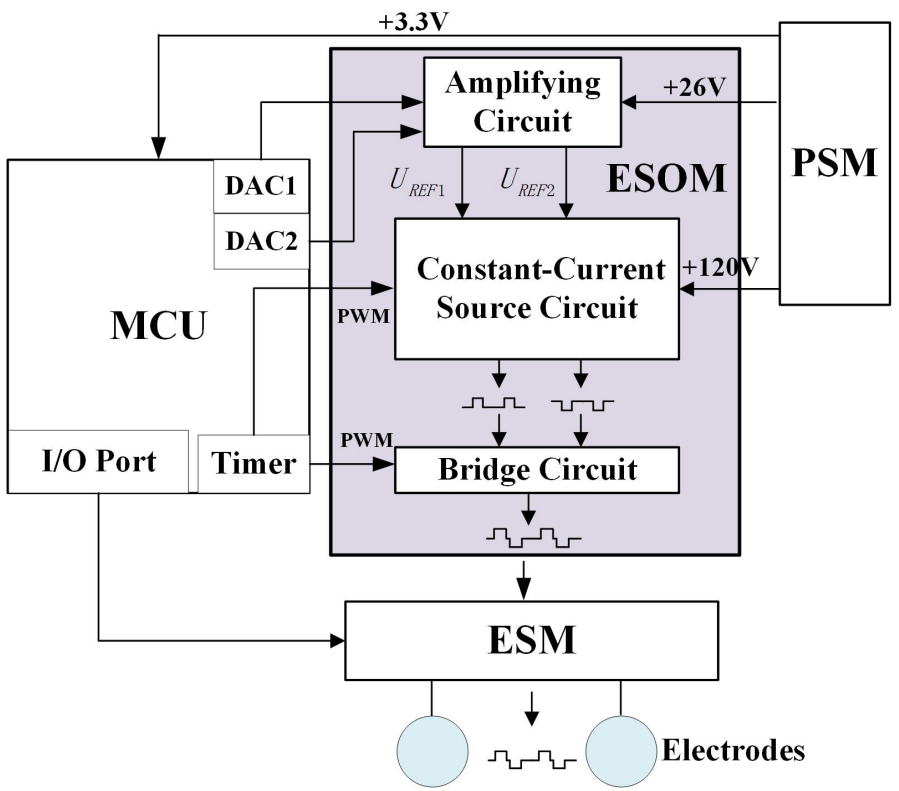

Fig. 5. The structure of stimulation current generating circuit.

vantage of making muscle contract consistently and repeatability with less variability in skin resistance [36]. In this study, we combine ESOM with MCU to generate the stimulation current and the circuit structure is shown in Fig. 5. The ESOM consists of three parts: amplifying circuit (AC), constant-curent source circuit (CCSC) and bridge circuit (BC). AC is a non-inverting operational amplifier circuit based on the operational amplifier LM358 (Texas Instruments), which is used to amplify the voltage of digital-to-analog converters (DACs) from MCU to be the reference voltage to control the positive and negative phase amplitude of the current respectively. CCSC is made up of two constant-current source, one is for generating the positive phase of the current and the other one for the negative phase. The schematic of the constant-current source circuit is shown in Fig. 6, which is based on the operational amplifier (LM358, Texas Instruments) and the MOSFET (FDD3N50NZ, Fairchild). And the magnitude of the stimulation current can be calculated by (6).

$$
I_{d}=\frac{R_{2} U_{R E F}}{\left(R_{2}+R_{3}\right) R_{s}}
$$

where $I_{d}$ is the stimulation current applied to skin (skin is the "LOAD" in Fig. 6), $U_{R E F}$ is the amplified DAC output from $\mathrm{MCU}$, and $R_{s}$ is the sampling resistance to capture the stimulation magnitude of current in real time.

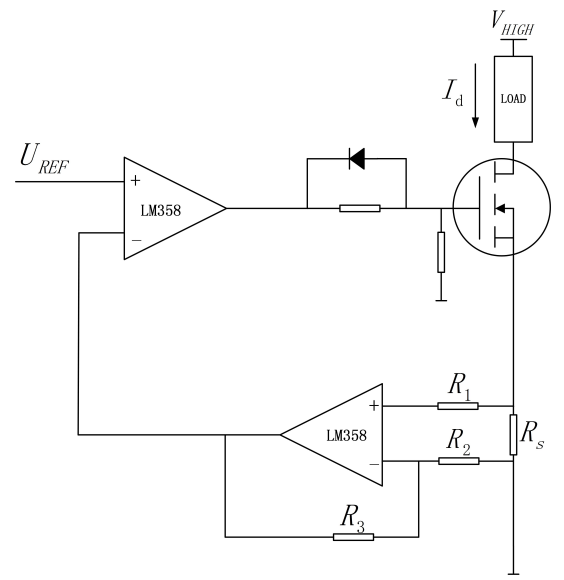

Fig. 6. Electrical schematic representation of constant-current source. The two LM358 make up of the feedback network for maintaining the constant current.

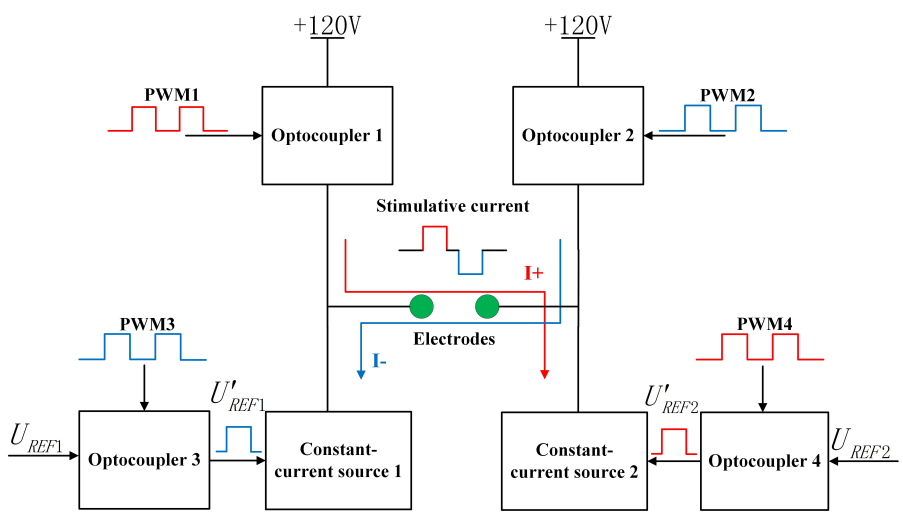

Fig. 7. Schematic representation of biphasic stimulation current generating in ESOM. The positive phase current I+ is generated when the constant-current source 2 works. The negative phase current I- is generated when the constantcurrent source 1 works.

Under the control of the PWM (Pulse-Width Modulation) signals from the timers in MCU, CCSC cooperates with BC 
to realize the biphasic square wave stimulation current with desired frequency and duration, as shown in Fig. 7. The timing signals should be isolated from the analog circuit of stimulation current because of the huge voltage difference between them. In this study, the optocouplers are used to accomplish the isolation and to be high speed switches between the batteryvoltage-boosted power supply $(+120 \mathrm{~V})$ and the constantcurrent source (e.g. Optocoupler 1 and Optocoupler 2 in Fig. 7 ) and between the reference voltage and the constant-current source (e.g. Optocoupler 3 and Optocoupler 4). The I+ and

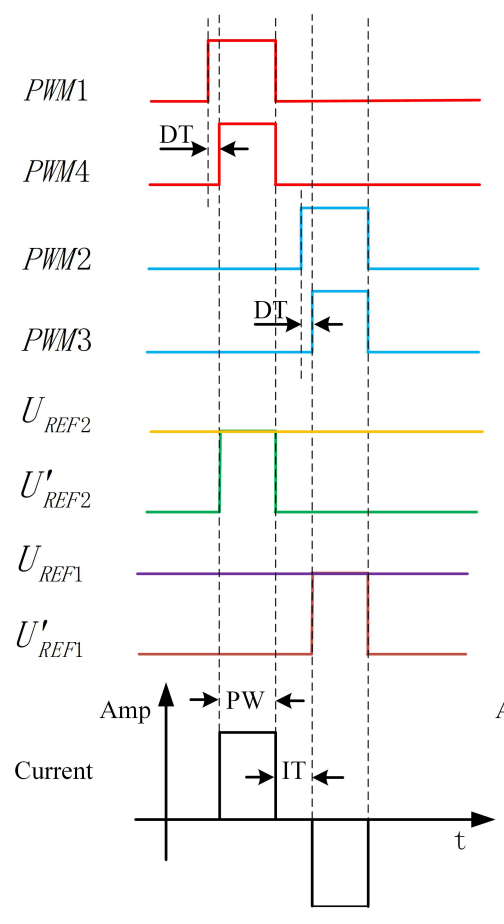

(a)

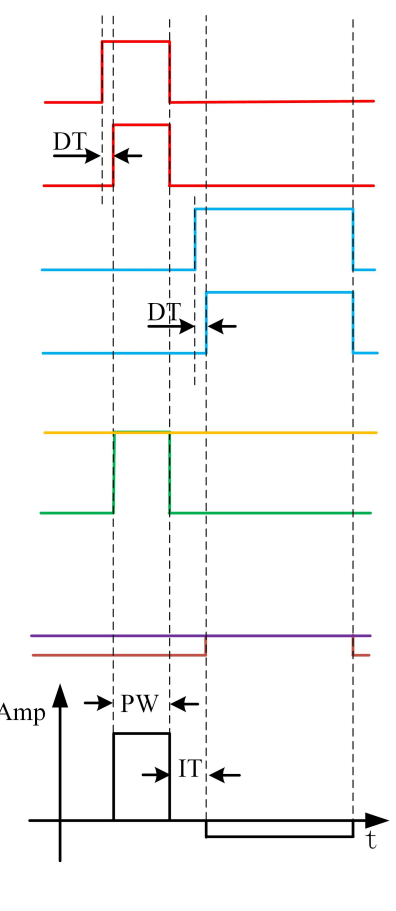

(b)
Fig. 8. The timing sequence diagram of generating biphasic stimulation current in ESOM: (a) Symmetric biphasic; (b) Asymmetric biphasic. DT represents dead-time, PW represents pulse width, Amp represents the current amplitude and IT is interval time. Note that the area of the positive pulse is equal to the area determined with the negative pulse in both kinds of the current wave form.

I- make up the biphasic stimulation current whose amplitude is determined by the reference voltage, while pulse width as well as frequency is determined by the PWM signals.

More details in time sequence for control signals, reference voltage and wave form are shown in Fig. 8. The overlapping parts of the high level between PWM1 and PWM4 mean that optocoupler 1 and optocoupler 4 are being switched on at the same time, which determines the pulse width of the positive phase current. Meanwhile $U_{R E F 2}^{\prime}$ is kept equal to $U_{R E F 2}$, making the constant-current source 2 work, to determine the amplitude of the current. The negative phase is generated similarly. All the PWM signals are running in the same frequency which is also the frequency of the stimulation current. When the pulse width of PWM3 and PWM4 are the same and the high level of $U_{R E F 1}^{\prime}$ and $U_{R E F 2}^{\prime}$ are equal in value, the symmetric biphasic pulse current is realized, and the asymmetric biphasic pulse current is realized when the pulse width of PWM3 and PWM4 are different, and meanwhile the high level of $U_{R E F 1}^{\prime}$ and $U_{R E F 2}^{\prime}$ are not equal.

No matter symmetric biphasic pulses or asymmetric biphasic pulses, the area of the negative phase should be equal to that of the positive phase, because the negative phase plays an important role in eliminating the charge accumulation in the skin and avoiding tissue damage. Due to the capacitance effect of the MOSFET in the constant-current source, a shorttime current fluctuation can be generated at the moment when the high voltage power supply is added to the constant-current source, which leads to the edge oscillation of the stimulation current pulse. Thus the purpose of the dead time DT (shown in Fig. 8) is to make the high voltage power supply linked to the constant-current a little earlier than the reference voltage. Then the constant-current is not working when the current fluctuation is happening, and it avoids the fluctuation being introduced into the stimulation current. In order to avoid the two constant-current sources working at the same time which will make the stimulation current into confusion, a short interval time (100 $\mu$ s) is set between PWM3 and PWM4, which results in an interval between the positive and negative phase.
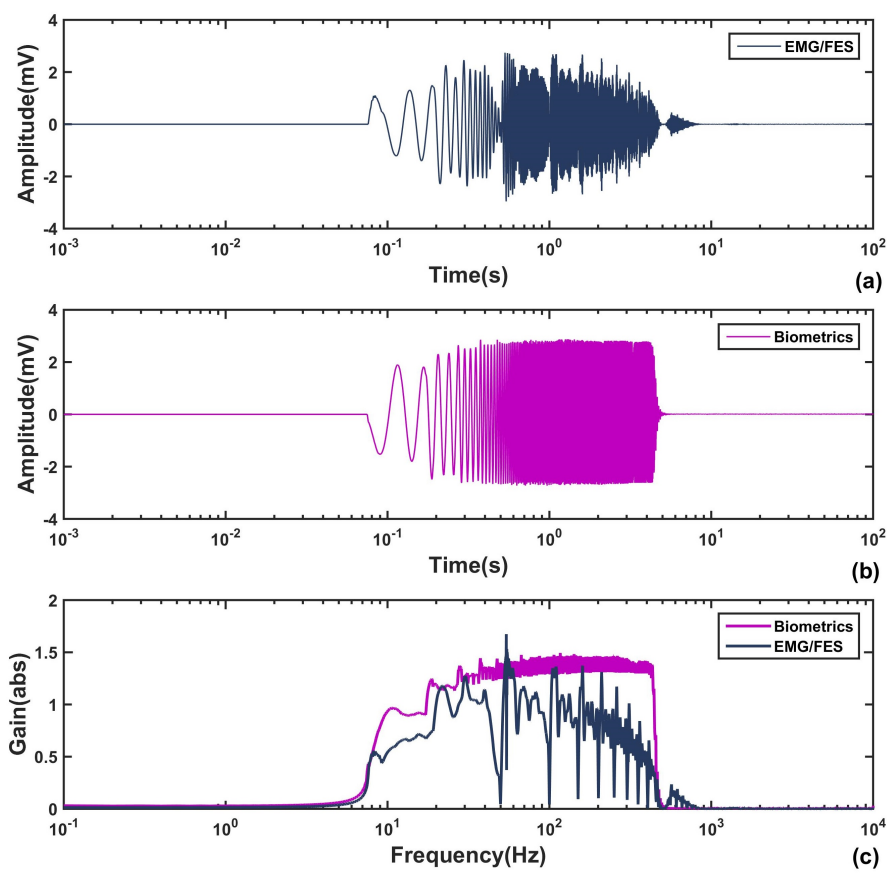

Fig. 9. Measurement results: (a) waveforms of sweeping signals detected by sEMG/FES integration system; (b) waveforms of sweeping signals detected by DataLOG MWX8 (Biometrics); (c) frequency response for sEMG/FES integration system and DataLOG MWX8 (Biometrics).

\section{SyStEM IMPLEMENTATION AND EVALUATION}

\section{A. EMG Processing Evaluation}

1) Frequency Response: A $4 \mathrm{mVpp}$ sinusoidal sweeping signal with its frequency sweeping linearly from $0.1 \mathrm{~Hz}$ to $10 \mathrm{kHz}$ was applied to the proposed system to measure the frequency response. The sweeping signal was generated by the Function/Arbitrary Waveform Generator (RIGOL DG1022U). 
The same protocol was repeated on DataLOG MWX8 (Biometrics Ltd, UK) for comparison. The test results are shown in Fig. 9. The EMG/FES integration system showed a comparable performance with DataLOG MWX8 in terms of amplifying the interested frequency components of sEMG signals $(20 \mathrm{~Hz}-$ $500 \mathrm{~Hz}$ ) while eliminating the unwanted signals. The sinking in frequency-response curve and the detected sweeping signals of the integration system is the result of the fourth order comb filter described in II-A.

2) Time-frequency Domain Characteristics: In this part, two commercial sEMG sensors DataLOGMWX8 (Biometrics Ltd, UK) and Trigno Wireless (Delsys Inc, USA) were involved to compare the time-frequency domain characteristics with the integration system. A healthy subject was asked to hold on hand close and hand open for $5 \mathrm{~s}$ in turn at a moderate level of effort according to the instructions on a computer screen, and the sEMG was recorded at the same time. The protocol was repeated on the same subject for testing different devices. And the electrodes/sensors were placed on the same muscle belly position of flexor carpi ulnaris (FCU) after cleaning with alcohol [37].
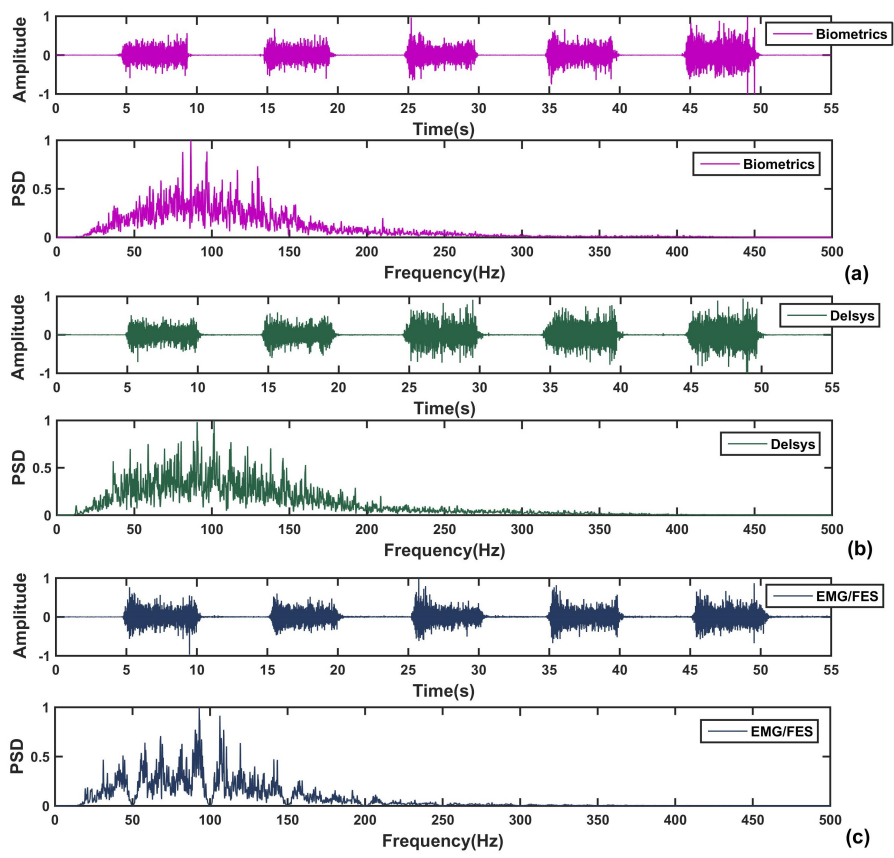

Fig. 10. Signals comparison between the proposed EMG/FES system and commecial sEMG acquisition systems in normalized sEMG signals and normalized PSD (power spectral densities) of the acquired sEMG signals: (a) Waveforms of Biometrics; (b) Waveforms of Delsys; (c) Waveforms of the proposed system.

As shown in Fig.10, the sEMG waveforms captured by different devices were closely similar to each other and all the devices could well detect EMG signals between $20 \mathrm{~Hz}$ and $350 \mathrm{~Hz}$; moreover, the PSD waveform for the sEMG captured by the designed system sinks obviously in $50 \mathrm{~Hz}$ and thereof compared to that of the two other systems, indicating the performance of removing the power line noise.

3) Signal-to-noise Ratio (SNR): In order to evaluate the signal quality of the integration system, SNR is compared among the integration system and the commercial devices mentioned before. A healthy subject with no nerve and limb disease took part in the experiment. During one trial, the subject was asked to hold on the handgrip dynamometer (Biometrics Ltd, UK) and keep the grip force at the level of $50 \%$ maximum volunteer contraction (MVC) with the vision feedback from computer for $10 \mathrm{~s}$ and then relax for $10 \mathrm{~s}$, meanwhile the sEMG signals were detected with the electrodes/sensors placed on the belly position of FCU muscle. The protocol was repeated on the same subject for testing different devices. The SNR was calculated by equation (7). According to the test result, the SNR of Trigno Wireless and DataLOG MWX8 are $28.4 \mathrm{~dB}$ and $25.7 \mathrm{~dB}$ respectively; the SNR of the integration system is $39.9 \mathrm{~dB}$. The signal quality of the integration system increased by $40.5 \%$ and $55.3 \%$ compared to the two commercial devices in the same testing environment.

$$
S N R=20 \log \frac{R M S_{\text {signal }}}{R M S_{\text {noise }}}
$$

\section{B. FES Outputting Evaluation}

1) Waveform of Stimulation Current: The parameters of the stimulation current consist of amplitude, pulse width and frequency. Fig. 11 shows the waveform of the stimulation current generated by the proposed system with different parameters. For Fig. 11(a), no DT was added to the control signals, so there were fluctuations (pointed by the red arrows) in the bipolar square wave; for Fig. 11(b), DT was only added to the control signals for generating the negative square wave so that fluctuation pointed by the green arrow is much smaller compared to that pointed by the red arrow, indicating that the DT in control signals described in II-B can effectively suppress the fluctuation; for Fig. 11(c)-11(f), DT was added to the control signals for generating the bipolar square wave. It indicates that the system can output normal symmetrical and asymmetrical bipolar square wave for stimulation, though there is still small fluctuation appearing upon the positive phase. The remain fluctuation, whose maximum amplitude and duration were reduced to $60 \mathrm{~mA}$ and $7 \mu$ s, took place when the DT was set as $30 \mu \mathrm{s}$.

2) Output capability: The FES current-output capability of the integration system were evaluated in this study. The amplitudes of the voltage on load resistance were measured when the fixed stimulation current is going through it. The load resistance varied from $200 \Omega \sim 3 \mathrm{k} \Omega$ (Mental oxide film resistance, power $5 \mathrm{~W}$, error $\pm 5 \%$ ). As shown in Fig. $12,30 \mathrm{~mA}$ and $60 \mathrm{~mA}$ as fixed current output were tested respectively. Compared with the simulated results calculated by $I \times R_{\text {load }}, 60 \mathrm{~mA}$-output $\left(R_{\text {load }}<2 \mathrm{k} \Omega\right)$ and $30 \mathrm{~mA}$-output are similar with the simulated ones, considering the error of the load resistance. When $R_{\text {load }}>2 \mathrm{k} \Omega$, the system could not maintain the $60 \mathrm{~mA}$-output and the current decreased with the resistance increasing. It is because the maximum value of the high voltage supply to the constant-current is $+120 \mathrm{~V}$ and the load voltage can not reach $+120 \mathrm{~V}$ because of the power loss in the system. The test results indicated that the output capability of the integration system was comparable with many commercial devices introduced in [35]. 

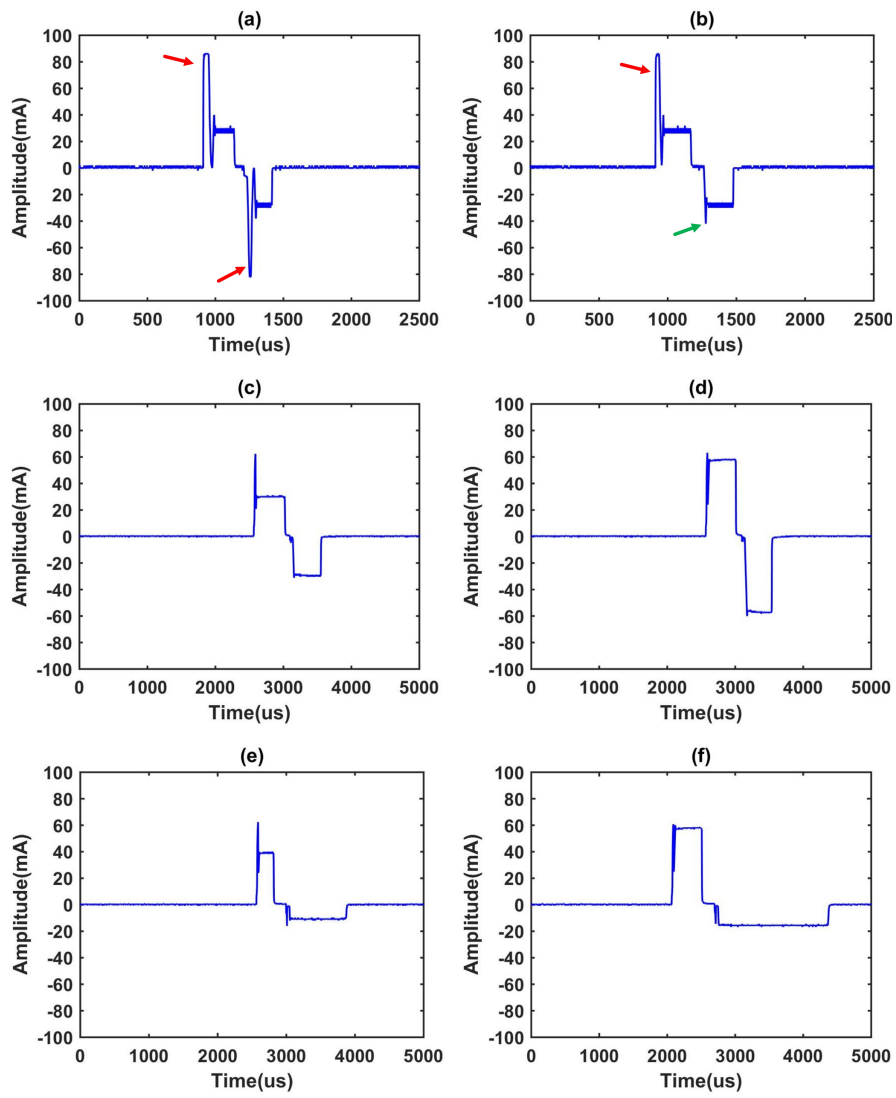

Fig. 11. Waveform of stimulation current with different parameters: (a) and

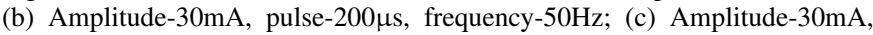
pulse- $400 \mu$ s, frequency- $50 \mathrm{~Hz}$; (d) Amplitude- $60 \mathrm{~mA}$, pulse- $400 \mu$ s, frequency-

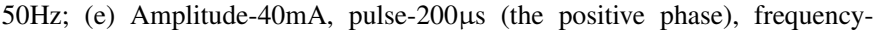

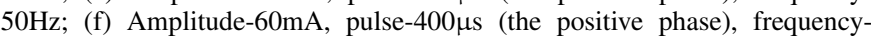
$50 \mathrm{~Hz}$. (a)-(d) are symmetrical bipolar square wave while (e)-(f) are asymmetrical bipolar square wave.

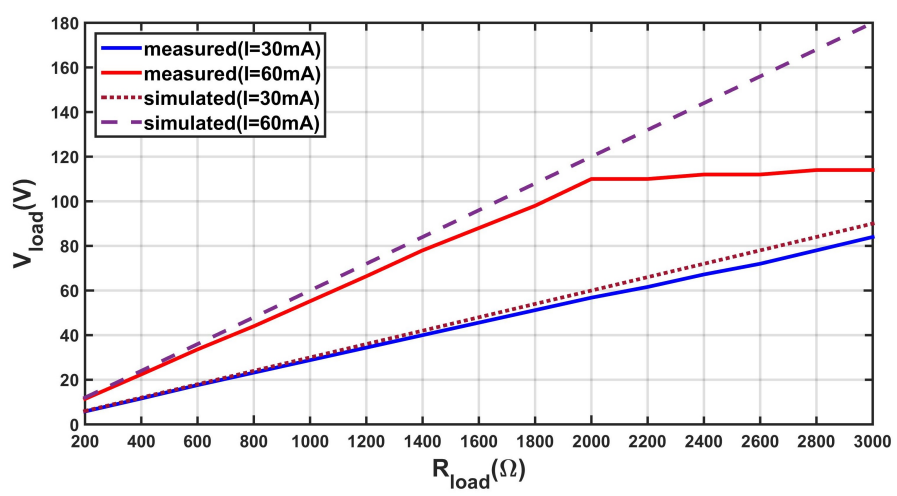

Fig. 12. The current output capability of the integration system. The stimulation current was set as $30 \mathrm{~mA}$ (the blue line) and $60 \mathrm{~mA}$ (the red line) when the load varied.

\section{EXPERIMENTS EVALUATION}

Preliminary experiment was conducted to verify the performance of the sEMG bias-driven FES. A healthy subject took part in the experiment, and the subject's right hand was used to simulate the affected hand. Because all the eight channels are equivalent to each other, here we choose channel one to be tested in the experiment. As shown in Fig. 13, a handgrip dynamometer (Biometrics Ltd, UK) was used to measure MVC force for the grasp of the subject. The experimental procedure was as follows. First, the subject was asked to perform maximum MVC handgrip contractions for five times using his "unaffected hand" (the left hand) with EMG signals of FCU stored. Two silica gel electrodes were placed on

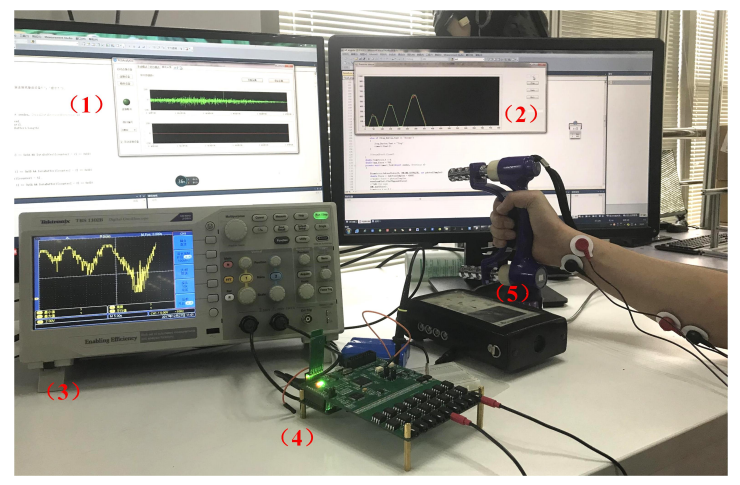

Fig. 13. The experiment for verifying controlling FES based on the EMG feedback in realtime using the integration system. (1) GUI for EMG demonstration; (2) GUI for force display; (3) Oscilloscope for stimulation intensity measurement; (4) The prototype; (5) The dynamometer. The EMG of FCU in the "affected hand" and the FES output intensity were measured in realtime while the "affected hand" performing grip with different force. The sEMG bias were generated every $200 \mathrm{~ms}, Q_{\max }$ and $Q_{\min }$ were $100 \%$ and $20 \%$ respectively, and $I_{\max }$ was set as $57 \mathrm{~mA}$.

the belly of FCU muscle and two were placed on the wrist for reference. Each contraction was kept for $5 \mathrm{~s}$ with 20 $\mathrm{s}$ rest between two consecutive contractions. For every $5 \mathrm{~s}$ contraction EMG signals, the data of the first second and the last second were discarded and the rest $3 \mathrm{~s}$ signals were used for EMG RMS value calculation for one contraction.

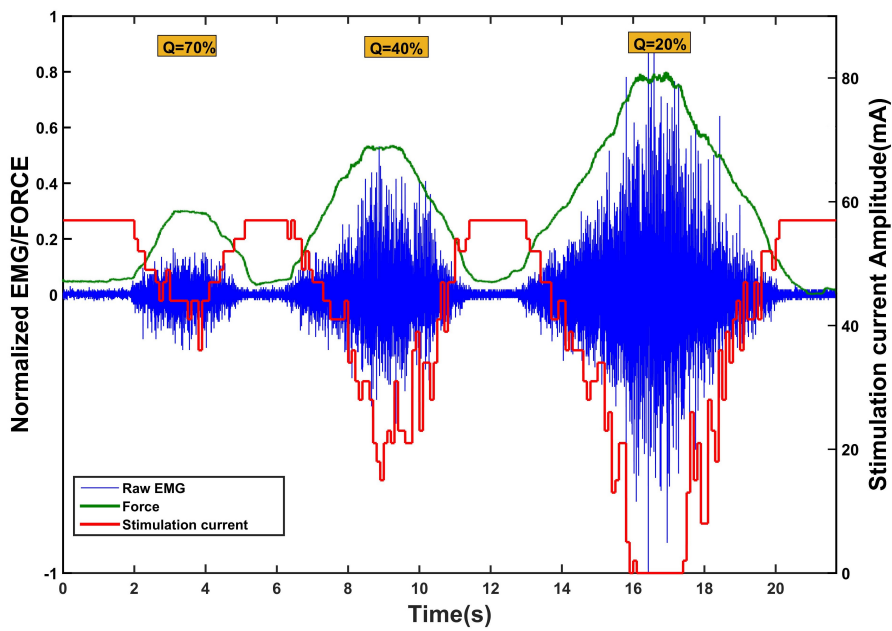

Fig. 14. The realtime FES output measurement based on the FCU EMG feedback when grip force varied. When $Q>20 \%$, the FES current amplitude changed from $0 \mathrm{~mA}$ to $I_{\max }$ and it was negative correlation with force and EMG intensity. When $Q<20 \%$, the amplitude of the current is $0 \mathrm{~mA}$. Because the EMG signals are non-stationary signals and the EMG processing segments were with no overlaps, the FES intensity changing was not smooth along time.

The mean value of the RMS for the five contractions was set as $R M S_{\text {unaffected }}$; second, the electrodes were moved and 
placed on the same position on the "affected hand" (the right hand), the subject performed the handgrip with his "affected hand" and controlled the grip force in realtime according to the visual feedback of the force value displayed on a computer screen in front of him. In order to avoid the effect of the FES current on controlling the handgrip force, the $U_{R E F}$ in equation (6) was measured to quantify the current to be applied rather than output the current to the skin directly.

Fig. 14 shows the result of the experiment. It indicated that the FES intensity can be well controlled according to the EMG feedback in real time. The weaker the EMG is, the higher the FES intensity will be output to the muscle. That means that weaker muscles in the affected hand will get more assistance from the integration system with higher FES, while the healthier muscles will get lower FES. Moreover, if the real-time $Q$ of the detected muscle is less than $Q_{\min }$ when the affected hand is performing a certain gesture, it means the muscle in the affected side is as strong as the symmetrical muscle in the unaffected side which can supply normal force to perform the gesture, so there will be no FES output for that muscle.

\section{DISCUSSION AND CONCLUSION}

The system evaluation in III has verified the validity of the proposed system for EMG processing and FES output respectively. It indicates that the system can effectively extract EMG signals and output controllable stimulation current. The experiment in IV has tested the performance of the closed-loop system. The FES output intensity was dynamic and changed according to the EMG feedback to make up for the strength defect of weak muscles. The prototype shows potential in biomedical and clinical applications for stroke rehabilitation.

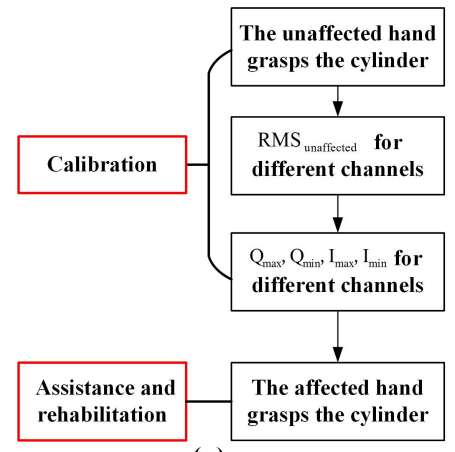

(a)

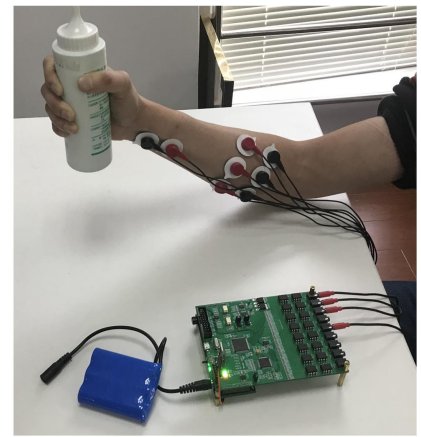

(b)
Fig. 15. The grasp training using the integration system: (a) The operation flow chat. (b) The working sketches.

In general, different muscles in forearm should work together to complete one hand motion, so more than one channel will work in a certain order with a small time interval between each other to assist the users in performing one hand motion. Fig. 15 shows an example of holding the cylinder aided by three channels of the system. Parameters such as $R M S_{\text {unaffected }}$, $Q_{\max }, Q_{\min }, I_{\max }$ and $I_{\min }$ for each channel should be calibrated respectively before applying the system to the affected hand. The system can act as an electrical prosthesis to assist the user who suffers from muscle weakness for motion training.

However, it should be noted that the proposed system can not evaluate the muscle state for users in flaccid paralysis period caused by severe stroke nor reduce symptoms for users with strong muscle spasticity. The patients with myasthenia in the affected side caused by mild stroke or in later period of stroke rehabilitation are the focus of this study.

Compared with traditional open-loop therapies, the proposed novel system integrated EMG processing and FES in hardware and software and could sense the user's active participation through EMG to train the neuromuscular system, which could increase the rehabilitation efficiency [12]. Different from those stimulators equipped with sensor interfaces mentioned in section I, the EMG feedback of the designed system could better reflect the physiological states of muscles than FSR, accelerometers and push buttons. To overcome the deficiency of uniform EMG evaluation standard for the affected hand, a novel evaluation method was proposed that the pathological states of the affected hand were quantified by the EMG bias between the affected and unaffected hand. That is to say, the EMG signals from the unaffected forearm were used as reference to evaluate the mirror symmetric muscle in the affected side forearm. Thus, different from the closed-loop FES studied in [28], [38], [39], which concentrated on the FES control strategies based on the FES-induced EMG rather than sensing the patients' neuromuscular pathological state, the FES in this study was driven by the EMG bias, so that it could not only avoid the unnecessary stimulation on the unaffected nerves and muscles to reduce the sufferings of users but also alleviate muscle fatigue compared to the cycling fixed intensity FES used in clinical. Furthermore, the designed system had advantages of wireless communication, multiple channels, portable size and real-time capability.

Extended functions can be developed in the designed system for different applications. It can be used as a multi-channel EMG acquisition system for EMG analysis and a normal multi-channel FES stimulator independently. When outputing small current, the system can be integrated with a dexterous hand prosthesis to setup the EMG controlling hand prosthesis based on electro-tactile feedback closed loop to improve the prosthesis performance [40], [41].

In conclusion, this paper presents the development of a sEMG bias-driven FES system for upper-limb stroke rehabilitation. The proposed system meets the design requirements for detecting motion intention, muscle states and has potential to realize active rehabilitation for stroke patients. To drive the proposed system into clinical application, future work has been planned as follows: 1) Optimizing the system parameters according to clinical trials; 2) Applying the system to stroke patients for clinical evaluation; 3) Research on human-machine interface based on EMG, ultrasound and haptic feedback using this system will also be involved.

\section{ACKNOWLEDGMENT}

The authors would like to thank all the participants in the experiments. The authors also appreciate Weichao Guo and 
Linjun Bao for their guidance in the study and during the preparation of this paper. Besides, the authors would like to thank for the cooperation project (SJTU-SD-FZ-Q2302A2016) between Elonxi Ltd and Shanghai Jiao Tong University.

\section{REFERENCES}

[1] W. H. Organization, The world health report 2003: shaping the future. World Health Organization, 2003.

[2] C. Warlow, J. Van Gijn, M. S. Dennis, J. M. Wardlaw, P. A. Sandercock, G. Rinkel, and P. Langhorne, "Stroke: practical management," 2008.

[3] F. Resquín, A. C. Gómez, J. Gonzalez-Vargas, F. Brunetti, D. Torricelli, F. M. Rueda, R. C. de la Cuerda, J. C. Miangolarra, and J. L. Pons, "Hybrid robotic systems for upper limb rehabilitation after stroke: A review," Medical engineering \& physics, vol. 38, no. 11, pp. 1279-1288, 2016.

[4] G. Yavuzer, R. Selles, N. Sezer, S. Sütbeyaz, J. B. Bussmann, F. Köseoğlu, M. B. Atay, and H. J. Stam, "Mirror therapy improves hand function in subacute stroke: a randomized controlled trial," Archives of physical medicine and rehabilitation, vol. 89, no. 3, pp. 393-398, 2008.

[5] B. French, L. Thomas, M. Leathley, C. Sutton, J. McAdam, A. Forster, P. Langhorne, C. Price, A. Walker, and C. Watkins, "Does repetitive task training improve functional activity after stroke? a cochrane systematic review and meta-analysis," Journal of rehabilitation medicine, vol. 42, no. 1 , pp. 9-15, 2010.

[6] F. Malouin, P. L. Jackson, and C. L. Richards, "Towards the integration of mental practice in rehabilitation programs. a critical review," Frontiers in human neuroscience, vol. 7, 2013.

[7] W. H. Chang and Y.-H. Kim, "Robot-assisted therapy in stroke rehabilitation," Journal of stroke, vol. 15, no. 3, p. 174, 2013.

[8] J. D. Schaechter, "Motor rehabilitation and brain plasticity after hemiparetic stroke," Progress in neurobiology, vol. 73, no. 1, pp. 61-72, 2004.

[9] D. Wade, R. Langton-Hewer, V. A. Wood, C. Skilbeck, and H. Ismail, "The hemiplegic arm after stroke: measurement and recovery." Journal of Neurology, Neurosurgery \& Psychiatry, vol. 46, no. 6, pp. 521-524, 1983.

[10] V. R. Edgerton and R. R. Roy, "Robotic training and spinal cord plasticity," Brain research bulletin, vol. 78, no. 1, pp. 4-12, 2009.

[11] M. Lotze, C. Braun, N. Birbaumer, S. Anders, and L. G. Cohen, "Motor learning elicited by voluntary drive," Brain, vol. 126, no. 4, pp. 866-872, 2003.

[12] C. T. Freeman, A.-M. Hughes, J. H. Burridge, P. H. Chappell, P. L. Lewin, and E. Rogers, "A model of the upper extremity using fes for stroke rehabilitation," Journal of biomechanical engineering, vol. 131, no. 3, p. 031011, 2009.

[13] D. Rushton, "Functional electrical stimulation and rehabilitationłan hypothesis," Medical engineering \& physics, vol. 25, no. 1, pp. 75-78, 2003.

[14] Y. Fang, N. Hettiarachchi, D. Zhou, and H. Liu, "Multi-modal sensing techniques for interfacing hand prostheses: a review," IEEE Sensors Journal, vol. 15, no. 11, pp. 6065-6076, 2015.

[15] C. L. Edwards, S. Sudhakar, M. T. Scales, K. L. Applegate, W. Webster, and R. H. Dunn, "Electromyographic (emg) biofeedback in the comprehensive treatment of central pain and ataxic tremor following thalamic stroke," Applied psychophysiology and biofeedback, vol. 25, no. 4, pp. 229-240, 2000.

[16] L. Dipietro, M. Ferraro, J. J. Palazzolo, H. I. Krebs, B. T. Volpe, and N. Hogan, "Customized interactive robotic treatment for stroke: Emg-triggered therapy," IEEE Transactions on Neural Systems and Rehabilitation Engineering, vol. 13, no. 3, pp. 325-334, 2005.

[17] X. Hu, K. Tong, R. Song, X. Zheng, K. Lui, W. Leung, S. Ng, and S. AuYeung, "Quantitative evaluation of motor functional recovery process in chronic stroke patients during robot-assisted wrist training," Journal of Electromyography and Kinesiology, vol. 19, no. 4, pp. 639-650, 2009.

[18] B. Cesqui, P. Tropea, S. Micera, and H. I. Krebs, "Emg-based pattern recognition approach in post stroke robot-aided rehabilitation: a feasibility study," Journal of neuroengineering and rehabilitation, vol. 10, no. 1 , p. $75,2013$.

[19] C. L. Lynch and M. R. Popovic, "Functional electrical stimulation," IEEE control systems, vol. 28, no. 2, pp. 40-50, 2008.

[20] W. Liberson, "Functional electrotherapy: stimulation of the peroneal nerve synchronized with the swing phase of the gait of hemiplegic patients," Arch Phys Med Rehabil, vol. 42, p. 101, 1961.
[21] D. B. Popović, "Advances in functional electrical stimulation (fes)," Journal of Electromyography and Kinesiology, vol. 24, no. 6, pp. 795802, 2014.

[22] S. M. Robbins, P. E. Houghton, M. G. Woodbury, and J. L. Brown, "The therapeutic effect of functional and transcutaneous electric stimulation on improving gait speed in stroke patients: a meta-analysis," Archives of physical medicine and rehabilitation, vol. 87, no. 6, pp. 853-859, 2006.

[23] S. K. Sabut, P. K. Lenka, R. Kumar, and M. Mahadevappa, "Effect of functional electrical stimulation on the effort and walking speed, surface electromyography activity, and metabolic responses in stroke subjects," Journal of Electromyography and Kinesiology, vol. 20, no. 6, pp. 11701177, 2010.

[24] G. Alon, A. F. Levitt, and P. A. McCarthy, "Functional electrical stimulation enhancement of upper extremity functional recovery during stroke rehabilitation: a pilot study," Neurorehabilitation and neural repair, vol. 21, no. 3, pp. 207-215, 2007.

[25] F. Quandt and F. C. Hummel, "The influence of functional electrical stimulation on hand motor recovery in stroke patients: a review," Experimental \& translational stroke medicine, vol. 6, no. 1, p. 9, 2014

[26] I. K. Hong, J. B. Choi, and J. H. Lee, "Cortical changes after mental imagery training combined with electromyography-triggered electrical stimulation in patients with chronic stroke," Stroke, vol. 43, no. 9, pp. 2506-2509, 2012

[27] T. Fujiwara, Y. Kasashima, K. Honaga, Y. Muraoka, T. Tsuji, R. Osu, K. Hase, Y. Masakado, and M. Liu, "Motor improvement and corticospinal modulation induced by hybrid assistive neuromuscular dynamic stimulation (hands) therapy in patients with chronic stroke," Neurorehabilitation and neural repair, vol. 23, no. 2, pp. 125-132, 2009.

[28] Q. Zhang, M. Hayashibe, and C. Azevedo-Coste, "Evoked electromyography-based closed-loop torque control in functional electrical stimulation," IEEE Transactions on Biomedical Engineering, vol. 60, no. 8, pp. 2299-2307, 2013.

[29] T. D. Scott, P. Peckham, and K. Kilgore, "Tri-state myoelectric control of bilateral upper extremity neuroprostheses for tetraplegic individuals," IEEE Transactions on Rehabilitation Engineering, vol. 4, no. 4, pp. 251-263, 1996

[30] E. Langzam, E. Isakov, and J. Mizrahi, "Evaluation of methods for extraction of the volitional emg in dynamic hybrid muscle activation," Journal of neuroengineering and rehabilitation, vol. 3, no. 1, p. 27, 2006.

[31] M. B. I. Reaz, M. S. Hussain, and F. Mohd-Yasin, "Emg analysis using wavelet functions to determine muscle contraction," in International Conference on E-Health Networking, Applications and Services, 2006. Healthcom, 2006, pp. 132-134.

[32] D. D. Koutsouris and A. A. Lazakidou, "Concepts and trends in healthcare information systems," British Journal of Psychology, vol. 69 , no. 1, pp. 25-35, 2014.

[33] Y. Fang, X. Zhu, and H. Liu, "Development of a surface emg acquisition system with novel electrodes configuration and signal representation," in International Conference on Intelligent Robotics and Applications. Springer, 2013, pp. 405-414.

[34] Y. Fang, H. Liu, G. Li, and X. Zhu, "A multichannel surface emg system for hand motion recognition," International Journal of Humanoid Robotics, vol. 12, no. 02, p. 1550011, 2015.

[35] B. J. Broderick, P. P. Breen, and G. OLaighin, "Electronic stimulators for surface neural prosthesis," Journal of automatic control, vol. 18, no. 2, pp. 25-33, 2008.

[36] L. R. Sheffler and J. Chae, "Neuromuscular electrical stimulation in neurorehabilitation," Muscle \& nerve, vol. 35, no. 5, pp. 562-590, 2007.

[37] E. Forvi, M. Bedoni, R. Carabalona, M. Soncini, P. Mazzoleni, F. Rizzo, C. OMahony, C. Morasso, D. G. Cassarà, and F. Gramatica, "Preliminary technological assessment of microneedles-based dry electrodes for biopotential monitoring in clinical examinations," Sensors and Actuators A: Physical, vol. 180, pp. 177-186, 2012.

[38] Z. Li, M. Hayashibe, D. Andreu, and D. Guiraud, "Real-time closedloop fes control of muscle activation with evoked emg feedback," in International Ieeelembs Conference on Neural Engineering, 2015, pp. 623-626.

[39] M. Hayashibe, "Evoked electromyographically controlled electrical stimulation," Frontiers in Neuroscience, vol. 10, no. 434, 2016.

[40] K. Li, Y. Fang, Y. Zhou, and H. Liu, "Non-invasive stimulation-based tactile sensation for upper-extremity prosthesis: a review," IEEE Sensors Journal, vol. 17, no. 9, pp. 2625-2635, 2017.

[41] Y. Fang, D. Zhou, K. Li, and H. Liu, "Interface prostheses with classifierfeedback based user training," IEEE Transactions on Biomedical Engineering, vol. PP, no. 99, pp. 1-1, 2016. 